\title{
Revisit Spectrum of Baryonium in Heavy Baryon Chiral Perturbation Theory
}

\author{
Yue-De Chen ${ }^{a *}$ Cong-Feng Qiao ${ }^{a \dagger}$, Peng-Nian Shen ${ }^{e, b, c \ddagger}$ and Zhuo-Quan Zeng ${ }^{d \S}$ \\ a) Department of Physics, University of the Chinese Academy of Sciences \\ YuQuan Road 19A, 100049, Beijing, China \\ b) Theoretical Physics Center for Science Facilities (TPCSF), CAS \\ YuQuan Road 19B, 100049, Beijing, China \\ c) Institute of High Energy Physics, CAS, Beijing, 100049, China \\ d) College of Physics and Electronic Engineering, Hainan Normal University, Haikou, 571158, China \\ e) College of Physics and Technology, Guangxi Normal University, Guilin 541004, China
}

\begin{abstract}
In the framework of the heavy baryon perturbation theory, in which the two-pion exchange is considered, the physical properties of heavy-baryon-anti-heavy-baryon systems are revisited. The potentials between heavy-baryon and anti-heavy-baryon are extracted in a holonomic form. Based on the extracted potentials, the s-wave scattering phase shifts and scattering lengths of $\Lambda_{c^{-}} \bar{\Lambda}_{c}$ and $\Sigma_{c^{-}} \bar{\Sigma}_{c}$ are calculated. From these scattering features, it is found that the $\Lambda_{c}-\bar{\Lambda}_{c}$ system can be bound only when the value of the coupling constant $g_{2}$ is larger than that from the decay data of the $\Sigma_{c}\left(\Sigma_{c}^{*}\right) \rightarrow \Lambda_{c} \pi$ process. The binding condition for the $\Sigma_{c}-\bar{\Sigma}_{c}$ system is also examined. The binding possibilities of these systems deduced from the scattering calculations are also checked by the bound state calculation and the binding energies are obtained if the system can be really bound. The binding possibility of the $\Lambda_{b}-\bar{\Lambda}_{b}$ system is investigated as well.
\end{abstract}

\footnotetext{
*Email: chenyuede@ucas.ac.cn

$\dagger$ Email: qiaocf@ucas.ac.cn

†Email: shenpn@ihep.ac.cn

$\S$ Email: zengzhuoquan@hotmail.com
} 


\section{Introduction}

Charmonium and bottomonium are important objects in studying strong interactions and structures of hadrons. In the past decade, many new hadron states with heavy flavors, such as $Y(4260), Y(4360), Y(4660), Z^{ \pm}(4430), Y_{b}(10890)$, and etc., have been found in the $e^{+} e^{-}$annihilation and the $B$ meson decay experiments by BABAR and Belle [1], in particular, $Z_{c}(3900)$ has been observed in the $e^{+} e^{-}$annihilation by BESIII and Belle [2, 3] recently. These new findings have attracted much attention on the structure of hadron all over the particle and nuclear physics societies [4, 5, 6, 7]. However, some of the states cannot be identified as a conventional quarkonium with heavy flavor, such as the charmonium or its excited state because of their abnormal quantum numbers, masses, decay modes and corresponding branching ratios in experiments. Therefore, as mentioned in our previous paper [8], to explain the peculiar data, many postulates for their structures have been proposed, but up to now, no definite conclusions could be drawn yet.

One of the striking pictures among the postulates is the baryonium with heavy flavor. In the extended heavy baryonium picture used in our previous paper [8, 9], an approximate $\mathrm{SU}(2)$ symmetry between $\Lambda_{c}$ and $\Sigma_{c}^{0}$ is assumed, and $\Lambda_{c}$ and $\Sigma_{c}^{0}$ are taken as the basis vectors in the two-dimensional "C-spin" representation, which is analogues to the isospin in the nucleon doublet case. Apparently, these basis vectors can form a "C-spin" triplet and a "C-spin" singlet [9]. The key point is to verify if a heavy baryon and a heavy antibaryon can really form a bound state, the baryonium, dynamically. The simplest way to achieve this aim is extracting a potential between heavy baryons by using a theory, for instance the so-called heavy baryon chiral perturbation theory (HBCPT) which can effectively provide a good description for the heavy baryon, and then solving the Schrödinger equation for the energy eigenvalue and consequently the mass spectrum.

In fact, in our previous investigation [8] we have studied the possibility of forming a heavy baryonium by using HBCPT. The result showed that there might exist a heavy baryonium as long as the adopted values of the coupling constant at the baryon-Goldstoneboson vertex and the cutoff parameter $\Lambda$ are, respectively, in the special ranges, although

the result is very sensitive to such values. Apparently, the strong parameter dependence 
is undesirable. Such a dependence might come from the inappropriate approximation in deriving potentials, for instance the premature truncation to the term with $1 / r^{5 / 2}$ in the asymptotic expressions of the potentials expanded in $\lambda[8,[10$. This is because that the contribution from the two-pion-exchange potential is short ranged, but the expansion of the potential function in $\lambda$ requires a relatively larger $r$. Although the dominant contribution of the $\lambda$ integral comes from small $\lambda$ values, the expansion converges extremely slow. Moreover, whether the physical value of the coupling constant, which can be extracted from relevant decay data, supports the existence of a heavy baryonium is still questionable and this problem should further be investigated carefully.

In this paper, we first re-derive the potential between $\Lambda_{c}\left(\Sigma_{c}\right)$ and $\bar{\Lambda}_{c}\left(\bar{\Sigma}_{c}\right)$ in a holonomic form rather than a truncated expansion in Ref. [8]. Then, we study the $\Lambda_{c}-\bar{\Lambda}_{c}\left(\Sigma_{c}-\bar{\Sigma}_{c}\right)$ scattering to get scattering characters, in particular those closely related to its binding feature. Based on the enlightenment from scattering information, we finally calculate the binding behavior of the system to confirm whether a heavy baryonium really exists. The paper is organized as follows. In Section 2, the formalism of HBCPT is briefly recalled. The two body interaction potentials in the $\Lambda_{c}^{+}-\bar{\Lambda}_{c}^{+}$and $\Sigma_{c}^{0}-\bar{\Sigma}_{c}^{0}$ systems are given in Section 3. In Section 4, the numerical results for the scattering information and the mass spectra of possible heavy baryonia are presented. And the summary is given in Section 5.

\section{A brief introduction to HBCPT}

As commonly adopted, symbol $q_{1} q_{2} Q$, where $q_{1(2)}$ represents the light quark, and $Q$ denotes the heavy quark, describes a heavy baryon which contains one heavy quark and two light quarks. Assuming that two light quarks form a pair of diquark, then in the flavor space, these three quarks can form a symmetric sextet and an antisymmetric triplet, i.e. $3 \otimes 3=6 \oplus \overline{3}$. Because the wave function of the hadron in the color space is totally antisymmetric, the wave function in the direct product space of orbit, flavor and spin must be symmetric. Consequently, for a ground state hadron, the wave function in the flavor and spin spaces should be symmetric since the orbital wave function is symmetric.

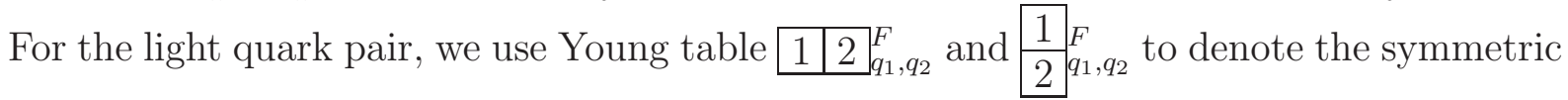




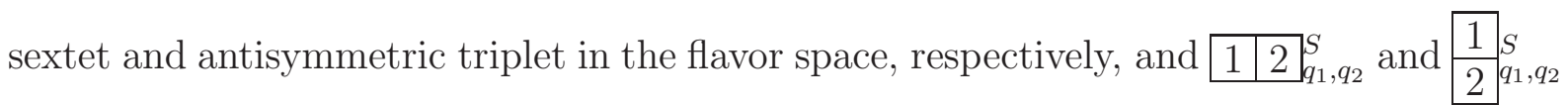
to represent the triplet and singlet in the spin space, respectively. Coupling these wave functions of a diquark to that of a heavy quark, denoted by $Q^{F} \times Q^{S}$, we have

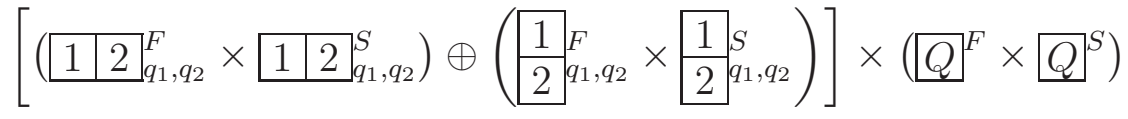

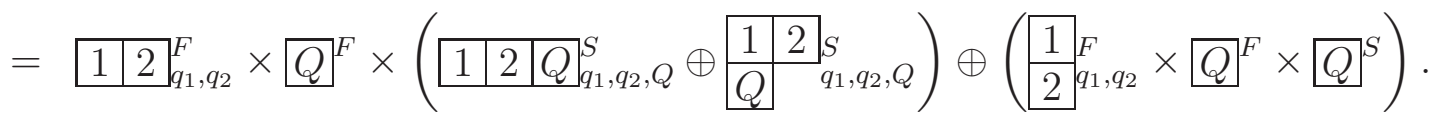

Equation (1) suggests that the sextet $\mathbf{6}$ has spin- $\frac{1}{2}$ and spin- $\frac{3}{2}$ states, while the triplet $\overline{\mathbf{3}}$ has only spin- $\frac{1}{2}$ states. Writing them explicitly in the matrix form, we have

$$
B_{6}=\left(\begin{array}{ccc}
\Sigma_{c}^{++} & \frac{1}{\sqrt{2}} \Sigma_{c}^{+} & \frac{1}{\sqrt{2}} \Xi_{c}^{\prime+} \\
\frac{1}{\sqrt{2}} \Sigma_{c}^{+} & \Sigma_{c}^{0} & \frac{1}{\sqrt{2}} \Xi_{c}^{\prime 0} \\
\frac{1}{\sqrt{2}} \Xi_{c}^{\prime+} & \frac{1}{\sqrt{2}} \Xi_{c}^{\prime 0} & \Omega_{c}^{0}
\end{array}\right)
$$

and

$$
B_{\overline{3}}=\left(\begin{array}{ccc}
0 & \Lambda_{c} & \Xi_{c}^{+} \\
-\Lambda_{c} & 0 & \Xi_{c}^{-} \\
-\Xi_{c}^{+} & -\Xi_{c}^{-} & 0
\end{array}\right)
$$

for the sextet and triplet of the charmed heavy baryon, respectively, and the same form of Eq.(2) for the spin- $\frac{3}{2} B_{6}^{*}$ multiple. These forms are also applicable to the bottomed heavy baryon multiple by substituting $c$ with $b$.

On the other hand, in terms of the chiral perturbation theory, one has the leading order vector and axial vector fields in $f_{\pi}$ [11, 12, 8]

$$
\begin{gathered}
V_{\mu}=\frac{1}{f_{\pi}^{2}} M \partial_{\mu} M, \\
A_{\mu}=-\frac{1}{f_{\pi}} \partial_{\mu} M,
\end{gathered}
$$

where $M$ is the Goldstone boson matrix

$$
M=\left(\begin{array}{ccc}
\frac{1}{\sqrt{2}} \pi^{0}+\frac{1}{\sqrt{6}} \eta & \pi^{+} & K^{+} \\
\pi^{-} & -\frac{1}{\sqrt{2}} \pi^{0}+\frac{1}{\sqrt{6}} \eta & K^{0} \\
K^{-} & \bar{K}^{0} & -\frac{2}{\sqrt{6}} \eta
\end{array}\right) .
$$


Then, the general form of the chiral-invariant Lagrangian can be written as [13]

$$
\begin{aligned}
\mathcal{L} & =\frac{1}{2} \operatorname{tr}\left[\bar{B}_{\overline{3}}\left(i \not D-M_{\overline{3}}\right) B_{\overline{3}}\right]+\operatorname{tr}\left[\bar{B}_{6}\left(i \not D-M_{6}\right) B_{6}\right] \\
& +\operatorname{tr}\left[\bar{B}_{6}^{* \mu}\left[-g_{\mu \nu}\left(i \not D-M_{6}^{*}\right)+i\left(\gamma_{\mu} D_{\nu}+\gamma_{\nu} D_{\mu}\right)-\gamma_{\mu}\left(i \not D+M_{6}^{*}\right) \gamma_{\nu}\right] B_{6}^{* \nu}\right] \\
& +g_{1} \operatorname{tr}\left(\bar{B}_{6} \gamma_{\mu} \gamma_{5} A^{\mu} B_{6}\right)+g_{2} \operatorname{tr}\left(\bar{B}_{6} \gamma_{\mu} \gamma_{5} A^{\mu} B_{\overline{3}}\right)+h . c . \\
& +g_{3} \operatorname{tr}\left(\bar{B}_{6 \mu}^{*} A^{\mu} B_{6}\right)+h . c .+g_{4} \operatorname{tr}\left(\bar{B}_{6 \mu}^{*} A^{\mu} B_{\overline{3}}\right)+h . c . \\
& +g_{5} \operatorname{tr}\left(\bar{B}_{6}^{\nu *} \gamma_{\mu} \gamma_{5} A^{\mu} B_{6 \nu}^{*}\right)+g_{6} \operatorname{tr}\left(\bar{B}_{\overline{3}} \gamma_{\mu} \gamma_{5} A^{\mu} B_{\overline{3}}\right)
\end{aligned}
$$

where the chiral covariant derivative $D_{\mu}$ satisfies

$$
\begin{aligned}
& D_{\mu} B_{6}=\partial_{\mu} B_{6}+V_{\mu} B_{6}+B_{6} V_{\mu}^{T}, \\
& D_{\mu} B_{\overline{3}}=\partial_{\mu} B_{\overline{3}}+V_{\mu} B_{\overline{3}}+B_{\overline{3}} V_{\mu}^{T} .
\end{aligned}
$$

According to the heavy quark symmetry, six coupling constants approximately obey following relations:

$$
g_{1}=\frac{2 \sqrt{3}}{3} g_{3}=-\frac{2}{3} g_{5}, \quad g_{2}=-\frac{\sqrt{3}}{3} g_{4}, \quad g_{6}=0
$$

thus, we have only two free parameters $g_{1}$ and $g_{2}$ in the numerical calculation [13].

\section{The formulation for fwo body scattering potential}

To derive the two body scattering kernel and further the potential, as carried out in Ref.[8], we follow the technique in Refs. [14, 15]. We first write down the scattering amplitude to get the interaction kernel, and then, make the non-relativistic reduction. Further making Fourier transformation, we obtain the potential in the configuration space. Then, acting the operators onto a considered channel, we finally obtain the potential for such a particular system. Again, in this continuation paper, we calculate the potentials in four $2 \pi$-exchange diagrams shown in Fig,1 


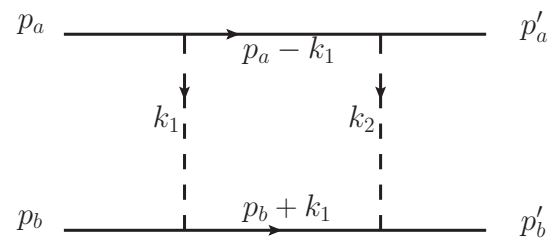

(a)

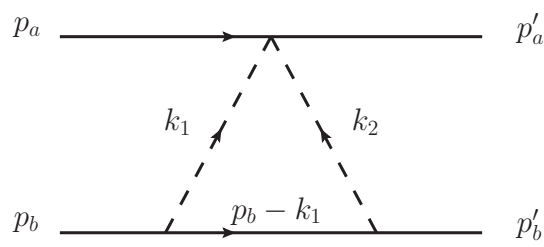

(c)

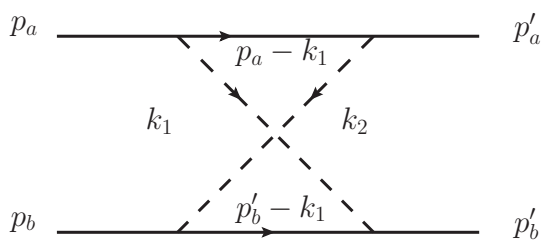

(b)

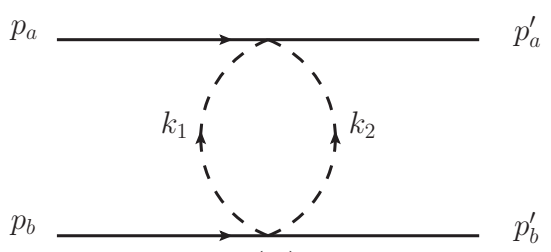

(d)

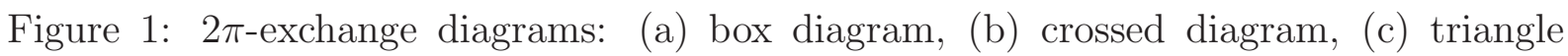
diagram, (d) two-pion loop diagram.

In the center of mass system (CMS), we define

$$
\begin{aligned}
\mathbf{p}_{a} & =-\mathbf{p}_{b}=\mathbf{p} \\
\mathbf{p}_{a}^{\prime} & =-\mathbf{p}_{b}^{\prime}=\mathbf{p}^{\prime} \\
P & =p_{a}+p_{b}=\left(E_{a}+E_{b}, 0\right)=(E, 0), \\
P^{\prime} & =p_{a}^{\prime}+p_{b}^{\prime}=\left(E_{a}^{\prime}+E_{b}^{\prime}, 0\right)=\left(E^{\prime}, 0\right), \\
p & =\frac{1}{2}\left(p_{a}-p_{b}\right)=(0, \mathbf{p}), \\
p^{\prime} & =\frac{1}{2}\left(p_{a}^{\prime}-p_{b}^{\prime}\right)=\left(0, \mathbf{p}^{\prime}\right),
\end{aligned}
$$

as the three-momenta of the initial and final states, the total four-momenta of the initial and final states, and the relative four-momenta of the initial and final states, respectively.

For the $\Lambda_{c}-\bar{\Lambda}_{c}$ interaction, we first calculate the potential in the box diagram. Following the prescription in Refs. [14, 15], we obtain the potential in the configuration space (detailed calculation can be found in Ref.[8])

$$
V_{B}\left(r_{1}, r_{2}\right)=-\left(\frac{g_{4}^{4}}{f_{\pi}^{4}}\right) \iint \frac{d^{3} \mathbf{k}_{1} d^{3} \mathbf{k}_{2}}{(2 \pi)^{6}} \frac{\mathcal{O}_{1}\left(\mathbf{k}_{1}, \mathbf{k}_{2}\right) e^{i \mathbf{k}_{1} \mathbf{r}_{1}} e^{i \mathbf{k}_{2} \mathbf{r}_{2}} f\left(\mathbf{k}_{1}^{2}\right) f\left(\mathbf{k}_{2}^{2}\right)}{2 E_{\mathbf{k}_{1}} E_{\mathbf{k}_{2}}\left(E_{\mathbf{k}_{1}}+\Delta\right)\left(E_{\mathbf{k}_{2}}+\Delta\right)\left(E_{\mathbf{k}_{1}}+E_{\mathbf{k}_{2}}\right)} .
$$

As commonly used, we take a Gaussian form for the form factor $f\left(\mathbf{k}_{i}^{2}\right)$ which regulates the integral. Further using the integral factorization technique, we get the non-local central 
potential

$$
\begin{aligned}
V_{B}\left(r_{1}, r_{2}\right)=- & \left(\frac{g_{4}^{4}}{f_{\pi}^{4}}\right) \frac{1}{\pi} \mathcal{O}_{1}\left(\mathbf{k}_{1}, \mathbf{k}_{2}\right)\left[\int_{0}^{\infty} \frac{d \lambda}{\Delta^{2}+\lambda^{2}} F\left(\lambda, r_{1}\right) F\left(\lambda, r_{2}\right)\right. \\
& \left.-\frac{2 \Delta}{\pi^{2}} \int_{0}^{\infty} \frac{d \lambda}{\Delta^{2}+\lambda^{2}} F\left(\lambda, r_{1}\right) \int_{0}^{\infty} \frac{d \lambda}{\Delta^{2}+\lambda^{2}} F\left(\lambda, r_{2}\right)\right]
\end{aligned}
$$

where $\Delta=M_{\Sigma_{c}^{\prime}}-M_{\Lambda_{c}}$ with $\Sigma_{c}^{\prime}$ being either $\Sigma_{c}^{+}$or $\Sigma_{c}^{+*}$ as the intermediate state in the $\Lambda_{c}^{+}-\bar{\Lambda}_{c}^{+}$interaction, and the form of $F(\lambda, r)$ can be found in Ref. [8].

In the same way, we can calculate potential in the crossed diagram and get the nonlocal potential

$$
V_{C}\left(r_{1}, r_{2}\right)=-\left(\frac{g_{4}^{4}}{f_{\pi}^{4}}\right) \frac{1}{\pi} \mathcal{O}_{1}\left(\mathbf{k}_{1}, \mathbf{k}_{2}\right) \int_{0}^{\infty} d \lambda \frac{\Delta^{2}-\lambda^{2}}{\left(\Delta^{2}+\lambda^{2}\right)^{2}} F\left(\lambda, r_{1}\right) F\left(\lambda, r_{2}\right) .
$$

Similarly, we obtain the non-local potential in the triangle diagram

$$
V_{\text {triangle }}\left(r_{1}, r_{2}\right)=\frac{g_{4}^{2}}{2 f_{\pi}^{4}} \iint \frac{d^{3} \mathbf{k}_{1} d^{3} \mathbf{k}_{2}}{(2 \pi)^{6}} \frac{\mathcal{O}_{2}\left(\mathbf{k}_{1}, \mathbf{k}_{2}\right)\left(E_{\mathbf{k}_{1}}+E_{\mathbf{k}_{2}}\right) e^{i \mathbf{k}_{1} \mathbf{r}_{1}} e^{i \mathbf{k}_{2} \mathbf{r}_{2}} f\left(\mathbf{k}_{1}^{2}\right) f\left(\mathbf{k}_{2}^{2}\right)}{E_{\mathbf{k}_{1}} E_{\mathbf{k}_{2}}\left(E_{\mathbf{k}_{1}}+\Delta\right)\left(E_{\mathbf{k}_{2}}+\Delta\right)},
$$

and potential in the $2 \pi$-loop diagram

$$
V_{2 \pi-\text { loop }}\left(r_{1}, r_{2}\right)=\frac{1}{16 f_{\pi}^{4}} \iint \frac{d^{3} \mathbf{k}_{1} d^{3} \mathbf{k}_{2}}{(2 \pi)^{6}} e^{i \mathbf{k}_{1} \mathbf{r}_{1}} e^{i \mathbf{k}_{2} \mathbf{r}_{2}} f\left(\mathbf{k}_{1}^{2}\right) f\left(\mathbf{k}_{2}^{2}\right) A
$$

where $E_{\mathbf{k}_{i}}=\sqrt{\mathbf{k}_{i}^{2}+m^{2}}$ and $A=-\frac{1}{2 E_{\mathbf{k}_{1}}}-\frac{1}{2 E_{\mathbf{k}_{2}}}+\frac{2}{E_{\mathbf{k}_{1}}+E_{\mathbf{k}_{2}}}$. In the above potentials, the operators $\mathcal{O}_{i}\left(\mathbf{k}_{1}, \mathbf{k}_{2}\right)$ with $i=1,2$ come from the non-relativistic reduction for the interactive vertices. Their general forms are

$$
\begin{aligned}
& \mathcal{O}_{1}\left(\mathbf{k}_{1}, \mathbf{k}_{2}\right)=c_{1}\left(\mathbf{k}_{1} \cdot \mathbf{k}_{2}\right)^{2}+c_{2}\left(\boldsymbol{\sigma}_{1} \cdot \mathbf{k}_{1} \times \mathbf{k}_{2}\right)\left(\boldsymbol{\sigma}_{2} \cdot \mathbf{k}_{1} \times \mathbf{k}_{2}\right), \\
& \mathcal{O}_{2}\left(\mathbf{k}_{1}, \mathbf{k}_{2}\right)=\left(\mathbf{k}_{1} \cdot \mathbf{k}_{2}\right) .
\end{aligned}
$$

Note that in the right side of Eq.(16), the first term will generate a central potential and the second term will produce a spin-spin potential and a tensor potential.

Finally, acting $\mathcal{O}_{i}\left(\mathbf{k}_{1}, \mathbf{k}_{2}\right)$ onto the concerned channel, making local approximation, and working out detailed derivation, for the box diagram, we obtain a central potential

$$
\begin{aligned}
V_{B C}(r)=- & {\left[\frac{1}{\pi} \int_{0}^{\infty} \frac{d \lambda}{\Delta^{2}+\lambda^{2}} F_{C}(\lambda, r)-\frac{4 \Delta}{\pi^{2} r^{2}}\left(\int_{0}^{\infty} \frac{d \lambda}{\Delta^{2}+\lambda^{2}} F^{\prime}(\lambda, r)\right)^{2}\right.} \\
& \left.-\frac{2 \Delta}{\pi^{2}}\left(\int_{0}^{\infty} \frac{d \lambda}{\Delta^{2}+\lambda^{2}} F^{\prime \prime}(\lambda, r)\right)^{2}\right]
\end{aligned}
$$


a spin-spin potential

$$
\begin{aligned}
V_{B S}(r)\left(\boldsymbol{\sigma}_{1} \cdot \boldsymbol{\sigma}_{2}\right)=- & {\left[\frac{2}{3 \pi} \int_{0}^{\infty} \frac{d \lambda}{\Delta^{2}+\lambda^{2}} F_{S}(\lambda, r)\right.} \\
& -\frac{4 \Delta}{3 \pi^{2}}\left(\frac{1}{r^{2}} \int_{0}^{\infty} \frac{d \lambda}{\Delta^{2}+\lambda^{2}} F^{\prime}(\lambda, r) \int_{0}^{\infty} \frac{d \beta}{\Delta^{2}+\beta^{2}} F^{\prime}(\beta, r)\right. \\
& \left.\left.+\frac{2}{r} \int_{0}^{\infty} \frac{d \lambda}{\Delta^{2}+\lambda^{2}} F^{\prime}(\lambda, r) \int_{0}^{\infty} \frac{d \beta}{\Delta^{2}+\beta^{2}} F^{\prime \prime}(\beta, r)\right)\right]\left(\boldsymbol{\sigma}_{1} \cdot \boldsymbol{\sigma}_{2} \gamma_{1}, 19\right)
\end{aligned}
$$

and a tensor potential

$$
\begin{aligned}
V_{B T}(r) S_{12}=- & {\left[\frac{2}{3 \pi} \int_{0}^{\infty} \frac{d \lambda}{\Delta^{2}+\lambda^{2}} F_{T}(\lambda, r)\right.} \\
& -\frac{4 \Delta}{3 \pi^{2}}\left(\frac{1}{r^{2}} \int_{0}^{\infty} \frac{d \lambda}{\Delta^{2}+\lambda^{2}} F^{\prime}(\lambda, r) \int_{0}^{\infty} \frac{d \beta}{\Delta^{2}+\beta^{2}} F^{\prime}(\beta, r)\right. \\
& \left.\left.-\frac{1}{r} \int_{0}^{\infty} \frac{d \lambda}{\Delta^{2}+\lambda^{2}} F^{\prime}(\lambda, r) \int_{0}^{\infty} \frac{d \beta}{\Delta^{2}+\beta^{2}} F^{\prime \prime}(\beta, r)\right)\right] S_{12} .
\end{aligned}
$$

For the crossed diagram, the central, spin-spin and tensor potentials are

$$
\begin{aligned}
& V_{C C}(r)=-\left[\frac{1}{\pi} \int_{0}^{\infty} d \lambda \frac{\Delta^{2}-\lambda^{2}}{\left(\Delta^{2}+\lambda^{2}\right)^{2}} F_{C}(\lambda, r)\right], \\
& V_{C S}(r)=-\left[\frac{1}{\pi} \int_{0}^{\infty} d \lambda \frac{\Delta^{2}-\lambda^{2}}{\left(\Delta^{2}+\lambda^{2}\right)^{2}} F_{S}(\lambda, r)\right],
\end{aligned}
$$

and

$$
V_{C T}(r)=-\left[\frac{1}{\pi} \int_{0}^{\infty} d \lambda \frac{\Delta^{2}-\lambda^{2}}{\left(\Delta^{2}+\lambda^{2}\right)^{2}} F_{T}(\lambda, r)\right],
$$

respectively, where $F_{C}, F_{S}$, and $F_{T}$ are

$$
\begin{aligned}
& F_{C}(\lambda, r)=\frac{2}{r^{2}} F^{\prime}(\lambda, r) F^{\prime}(\lambda, r)+F^{\prime \prime}(\lambda, r) F^{\prime \prime}(\lambda, r), \\
& F_{S}(\lambda, r)=\frac{F^{\prime}(\lambda, r)}{r}\left(\frac{F^{\prime}(\lambda, r)}{r}+2 F^{\prime \prime}(\lambda, r)\right), \\
& F_{T}(\lambda, r)=\frac{F^{\prime}(\lambda, r)}{r}\left(\frac{F^{\prime}(\lambda, r)}{r}-F^{\prime \prime}(\lambda, r)\right),
\end{aligned}
$$

respectively.

For the triangle diagram, at the order of $\mathcal{O}\left(\frac{1}{M_{H}}\right)$, we have only a central potential

$$
V_{T C}(r)=\frac{4 \Delta}{\pi^{2}} \int_{0}^{\infty} d \lambda \frac{\lambda^{2}}{\Delta^{2}+\lambda^{2}} F^{\prime}(\lambda, r) \int_{0}^{\infty} \frac{d \lambda}{\Delta^{2}+\lambda^{2}} F^{\prime}(\lambda, r) .
$$


Similarly, for the $2 \pi$-loop diagram, only a central potential contributes

$$
V_{2 \pi-\text { loop }}(r)=-\frac{2}{\pi}\left[\int_{0}^{\infty} d \lambda F(\lambda, r)\left(\frac{\Lambda^{3}}{8 \pi^{3 / 2}} \exp \left(-\frac{1}{4} \Lambda^{2} r^{2}\right)-2 \lambda^{2} F(\lambda, r)\right)\right] .
$$

Summing up all the potentials, we eventually obtain the two-pion-exchange potential for the heavy-baryon-anti-heavy-baryon interaction

$$
V(r)=V_{C}(r)+V_{S}(r) \boldsymbol{\sigma}_{1} \cdot \boldsymbol{\sigma}_{2}+V_{T}(r) \mathbf{S}_{12}
$$

where $V_{C}(r), V_{S}(r)$ and $V_{T}(r)$ are the radial parts of the central, spin-spin and tensor

potentials, respectively. From above potential forms, we see that the longest range of the obtained potentials is, as expected, that of the two-pion-exchange, because they have a quadratic product of $F(\lambda, r)$ (or derivatives), and thus have their longest range terms proportional to $\exp (-2 m r)$. In addition, we would point out that because the kernels in different channel are the same except the coefficients, for simplicity, we can derive the kernel itself first, and then add the coefficient later for the particular system.

Now, we go to specific systems.

\section{$3.1 \Lambda_{c}^{+}-\bar{\Lambda}_{c}^{+}$potential}

In the $\Lambda_{c}^{+}-\bar{\Lambda}_{c}^{+}$interaction, we assume that both $\Sigma_{c}^{+}$and $\Sigma_{c}^{+*}$ could be the intermediate state. The Lagrangian for spin- $\frac{1}{2} \Sigma_{c}-\pi-\Lambda_{c}$ interaction reads

$$
\mathcal{L}_{\Sigma_{c}-\pi-\Lambda_{c}}=-\frac{g_{2}}{f_{\pi}} \bar{\Sigma}_{c}^{++,+, 0} \gamma^{\mu} \gamma_{5} \partial_{\mu} \pi^{+, 0,-} \Lambda_{c}^{+}+\text {h.c. }
$$

where the strong coupling constant $g_{2}$ can be extracted from $\Sigma_{c}^{++} \rightarrow \Lambda_{c}^{+}+\pi^{+}$decay process (see Fig 3) by

$$
\Gamma=\frac{g_{2}^{2}|\mathbf{k}|}{8 f_{\pi}^{2} M_{\Sigma_{c}^{++}}^{2}}\left(M_{\Sigma_{c}^{++}}^{2}+M_{\Lambda_{c}^{+}}^{2}\right)\left[\left(M_{\Sigma_{c}^{++}}-M_{\Lambda_{c}^{+}}\right)^{2}-m_{\pi}^{2}\right],
$$

where $|\mathbf{k}|=94 \mathrm{MeV}$ is the momentum of the pion in the $\Sigma_{c}^{++}$rest frame, $f_{\pi}=0.132 \mathrm{GeV}$, $M_{\Sigma_{C}^{++}}=0.245 \mathrm{GeV}, M_{\Lambda_{c}^{+}}=0.229 \mathrm{GeV}$, and $\Gamma=2.23 \pm 0.30 \mathrm{MeV}$ [16]. The resultant phenomenological coupling constant is $g_{2}=0.5 \pm 0.07$. 


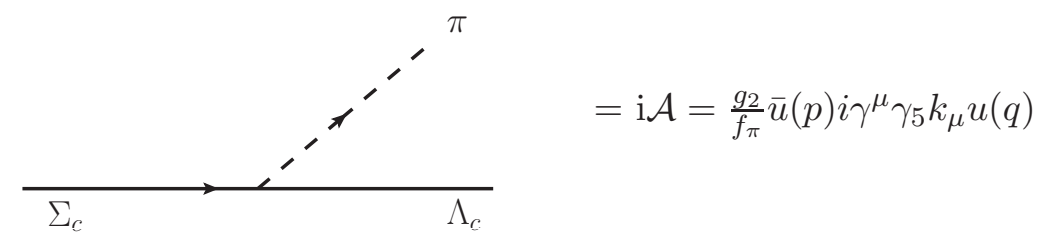

Figure 2: Vertex of the $\Sigma_{c}^{*}-\pi-\Lambda_{c}$ interaction for extracting $g_{2}$.

Based on the Lagrangian in Eq.(30), we have an explicit form of $\mathcal{O}_{1}\left(\mathbf{k}_{1}, \mathbf{k}_{2}\right)$ for both box and crossed diagrams

$$
\mathcal{O}_{1}\left(\mathbf{k}_{1}, \mathbf{k}_{2}\right)=\left(\mathbf{k}_{1} \cdot \mathbf{k}_{2}\right)^{2}+\left(\boldsymbol{\sigma}_{1} \cdot \mathbf{k}_{1} \times \mathbf{k}_{2}\right)\left(\boldsymbol{\sigma}_{2} \cdot \mathbf{k}_{1} \times \mathbf{k}_{2}\right) .
$$

It leads to a $\Lambda_{c}^{+}-\bar{\Lambda}_{c}^{+}$potential, caused by the $2 \pi$-exchange with $\Sigma_{c}$ as the intermediate state,

$$
\begin{aligned}
V_{1 \Lambda_{c}^{+} \bar{\Lambda}_{c}^{+}}(r) & =\frac{g_{2}^{4}}{f_{\pi}^{4}}\left[V_{B C}(r)+V_{C C}(r)+V_{T C}(r)\right]+\frac{1}{f_{\pi}^{4}} V_{2 \pi-l o o p}(r) \\
& +\frac{g_{2}^{4}}{f_{\pi}^{4}}\left[V_{B S}(r)+V_{C S}(r)\right]\left(\boldsymbol{\sigma}_{1} \cdot \boldsymbol{\sigma}_{2}\right)+\frac{g_{2}^{4}}{f_{\pi}^{4}}\left[V_{B T}(r)+V_{C T}(r)\right] S_{12} .
\end{aligned}
$$

The Lagrangian for spin- $\frac{3}{2} \Sigma_{c}^{*}-\pi-\Lambda_{c}$ interaction can be written as

$$
\mathcal{L}_{\Sigma_{c}^{*}-\pi-\Lambda_{c}}=-\frac{g_{4}}{f_{\pi}}\left(\bar{\Sigma}_{c}^{++},+, 0\right)^{* \mu} \partial_{\mu} \pi^{+, 0,-} \Lambda_{c}^{+}+\text {h.c. } .
$$

Similarly, the coupling constant $g_{4}$ can be extracted from the $\Sigma_{c}^{*} \rightarrow \Lambda_{c}+\pi$ decay process by

$$
\Gamma=\frac{g_{4}^{2}|\mathbf{k}| M_{\Sigma_{c}^{*}}^{2}}{96 f_{\pi}^{2}}\left[\left(1-\frac{M_{\Lambda_{c}}}{M_{\Sigma_{c}^{*}}}\right)^{2}-\frac{m_{\pi}^{2}}{M_{\Sigma_{c}^{*}}^{2}}\right]\left[\left(1+\frac{M_{\Lambda_{c}}}{M_{\Sigma_{c}^{*}}}\right)^{2}-\frac{m_{\pi}^{2}}{M_{\Sigma_{c}^{*}}^{2}}\right]^{2},
$$

with $|\mathbf{k}|=180 \mathrm{MeV}$ being the momentum of the pion in the rest frame of $\Sigma_{c}^{++*}, M_{\Sigma_{C}^{+*}}=$ $0.252 \mathrm{GeV}, M_{\Lambda_{c}^{0 *}}=0.229 \mathrm{GeV}$, and $\Gamma=14.9 \pm 1.9 \mathrm{MeV}$ [16]. The obtained coupling constant is $g_{4}=0.57 \pm 0.07$. Apparently, resultant $g_{4}$ and $g_{2}$ are not agreed with the symmetry relation shown in Eq.(10). This implies that the heavy quark symmetry is broken.

Similar to the above case, using Lagrangian in Eq.(34) we can explicitly write out $\mathcal{O}_{1}\left(\mathbf{k}_{1}, \mathbf{k}_{2}\right)$ for the box diagram as

$$
\mathcal{O}_{1}\left(\mathbf{k}_{1}, \mathbf{k}_{2}\right)=\frac{4}{9}\left(\mathbf{k}_{1} \cdot \mathbf{k}_{2}\right)^{2}-\frac{1}{9}\left(\boldsymbol{\sigma}_{1} \cdot \mathbf{k}_{1} \times \mathbf{k}_{2}\right)\left(\boldsymbol{\sigma}_{2} \cdot \mathbf{k}_{1} \times \mathbf{k}_{2}\right)
$$




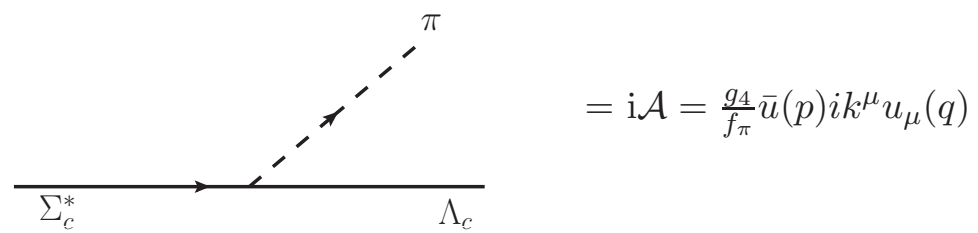

Figure 3: Vertex of the $\Sigma_{c}^{*}-\pi-\Lambda_{c}$ interaction for extracting $g_{4}$.

and for the crossed diagram as

$$
\mathcal{O}_{1}\left(\mathbf{k}_{1}, \mathbf{k}_{2}\right)=\frac{4}{9}\left(\mathbf{k}_{1} \cdot \mathbf{k}_{2}\right)^{2}+\frac{1}{9}\left(\boldsymbol{\sigma}_{1} \cdot \mathbf{k}_{1} \times \mathbf{k}_{2}\right)\left(\boldsymbol{\sigma}_{2} \cdot \mathbf{k}_{1} \times \mathbf{k}_{2}\right) .
$$

These lead to a $\Lambda_{c}^{+}-\bar{\Lambda}_{c}^{+}$potential, caused by the $2 \pi$-exchange with $\Sigma_{c}^{*}$ as the intermediate state,

$$
\begin{aligned}
V_{2 \Lambda_{c}^{+} \bar{\Lambda}_{c}^{+}}(r) & =\frac{4 g_{4}^{4}}{9 f_{\pi}^{4}}\left[V_{B C}(r)+V_{C C}(r)\right]+\frac{2 g_{4}^{2}}{3 f_{\pi}^{2}} V_{T C}(r) \\
& +\frac{g_{4}^{4}}{9 f_{\pi}^{4}}\left[-V_{B S}(r)+V_{C S}(r)\right]\left(\boldsymbol{\sigma}_{1} \cdot \boldsymbol{\sigma}_{2}\right)+\frac{g_{4}^{4}}{9 f_{\pi}^{4}}\left[-V_{B T}(r)+V_{C T}(r)\right] S_{12} .
\end{aligned}
$$

Putting these contributions together, we finally obtain the $\Lambda_{c}^{+} \bar{\Lambda}_{c}^{+}$potential

$$
V_{\Lambda_{c}^{+} \bar{\Lambda}_{c}^{+}}(r)=V_{1 \Lambda_{c}^{+} \bar{\Lambda}_{c}^{+}}(r)+V_{2 \Lambda_{c}^{+} \bar{\Lambda}_{c}^{+}}(r) .
$$

\section{$3.2 \Sigma_{c}^{0}-\bar{\Sigma}_{c}^{0}$ potential}

In the $\Sigma_{c}^{0}-\bar{\Sigma}_{c}^{0}$ interaction, both one-pion-exchange and two-pion-exchange are allowed. In the one-pion-exchange case, the Lagrangian of the $\Sigma_{c}^{0}-\pi-\Sigma_{c}^{0}$ interaction can be written as

$$
\mathcal{L}_{\Sigma_{c}^{0}-\pi-\Sigma_{c}^{0}}=-\frac{g_{1}}{\sqrt{2} f_{\pi}} \bar{\Sigma}_{c}{ }^{0} \gamma^{\mu} \gamma_{5} \partial_{\mu} \pi^{0,-} \Sigma_{c}^{0,+} .
$$

The axial current interaction (one-pion-exchange) causes a spin-spin potential

$$
V_{O P S}(r)\left(\boldsymbol{\sigma}_{1} \cdot \boldsymbol{\sigma}_{2}\right)=-\frac{g_{1}^{2}}{3 f_{\pi}^{2}}\left[I^{\prime \prime}(m, r)+\frac{1}{r} I^{\prime}(m, r)\right]\left(\boldsymbol{\sigma}_{1} \cdot \boldsymbol{\sigma}_{2}\right),
$$

and a tensor potential

$$
V_{O P T}(r) S_{12}=-\frac{g_{1}^{2}}{3 f_{\pi}^{2}}\left[I^{\prime \prime}(m, r)-\frac{1}{r} I^{\prime}(m, r)\right] S_{12},
$$


where the function $I(m, r)$ is given in the Appendix of Ref. [8]. Then we have one-pionexchange caused potential

$$
V_{1 \Sigma_{c}^{0} \bar{\Sigma}_{c}^{0}}(r)=V_{O P S}(r)\left(\boldsymbol{\sigma}_{1} \cdot \boldsymbol{\sigma}_{2}\right)+V_{O P T}(r) S_{12}
$$

In the two-pion-exchange case, both spin- $\frac{1}{2} \Sigma_{c}^{0}$ and $\Lambda_{c}^{+}$can be the intermediate state. So, we have the spin- $\frac{1}{2}$ intermediate state caused potential

$$
\begin{aligned}
V_{2 \Sigma_{c}^{0} \bar{\Sigma}_{c}^{0}}(r) & =\left[\left(\frac{g_{1}^{4}}{4 f_{\pi}^{4}}+\frac{g_{2}^{4}}{f_{\pi}^{4}}\right)\left(V_{B C}(r)+V_{C C}(r)\right)+\left(\frac{g_{1}^{2}}{2 f_{\pi}^{4}}+\frac{g_{2}^{2}}{f_{\pi}^{4}}\right) V_{T C}(r)+\frac{1}{f_{\pi}^{4}} V_{2 \pi-\text { loop }}(r)\right] \\
& +\left(\frac{g_{1}^{4}}{4 f_{\pi}^{4}}+\frac{g_{2}^{4}}{f_{\pi}^{4}}\right)\left[V_{B S}(r)+V_{C S}(r)\right]\left(\boldsymbol{\sigma}_{1} \cdot \boldsymbol{\sigma}_{2}\right) \\
& +\left(\frac{g_{1}^{4}}{4 f_{\pi}^{4}}+\frac{g_{2}^{4}}{f_{\pi}^{4}}\right)\left[V_{B T}(r)+V_{C T}(r)\right] S_{12}
\end{aligned}
$$

where $g_{1}$ stands for the coupling constant in the case where spin- $\frac{1}{2} \Sigma_{c}^{0}$ is an intermediate state.

Moreover, spin- $\frac{3}{2} \Sigma_{c}^{*}$, as an intermediate state, would also contributes. The Lagrangian of the $\Sigma_{c}^{0 *}-\pi-\Sigma_{c}^{0}$ interaction reads

$$
\mathcal{L}_{\Sigma_{c}^{0 *}-\pi-\Sigma_{c}^{0}}=-\frac{g_{3}}{\sqrt{2} f_{\pi}} \bar{\Sigma}^{0 * \mu} \partial_{\mu} \pi^{0} \Sigma^{0}
$$

with $g_{3}$ being the coupling constant. Based on this Lagrangian, following the same procedure used above, we have the spin- $\frac{3}{2} \Sigma_{c}^{*}$, as an intermediate state, caused potential

$$
\begin{aligned}
V_{3 \Sigma_{c}^{0} \bar{\Sigma}_{c}^{0}}(r) & =\frac{g_{3}^{4}}{9 f_{\pi}^{4}}\left(V_{B C}(r)+V_{C C}(r)\right)+\frac{g_{3}^{2}}{3 f_{\pi}^{4}} V_{T C}+\frac{g_{3}^{4}}{36 f_{\pi}^{4}}\left[-V_{B S}(r)+V_{C S}(r)\right]\left(\boldsymbol{\sigma}_{1} \cdot \boldsymbol{\sigma}_{2}\right) \\
& +\frac{g_{3}^{4}}{36 f_{\pi}^{4}}\left[-V_{B T}(r)+V_{C T}(r)\right] S_{12}
\end{aligned}
$$

Finally, we obtain the $\Sigma_{c}^{0}-\bar{\Sigma}_{c}^{0}$ potential

$$
V_{\Sigma_{c}^{0}-\bar{\Sigma}_{c}^{0}}(r)=V_{1 \Sigma_{c}^{0} \bar{\Sigma}_{c}^{0}}(r)+V_{2 \Sigma_{c}^{0} \bar{\Sigma}_{c}^{0}}(r)+V_{3 \Sigma_{c}^{0} \bar{\Sigma}_{c}^{0}}(r)
$$

\section{3 $\Lambda_{b}^{0}-\bar{\Lambda}_{b}^{0}$ potential}

The same formulas can also be applied to the $\Lambda_{b}^{0}-\bar{\Lambda}_{b}^{0}$ interaction except the $c$-flavored heavy baryon (antibaryon) is replaced by the $b$-flavored heavy baryon (antibaryon). 


\section{Numerical Result and Discussion}

In the numerical calculation, we take $m_{\pi}=0.135 \mathrm{GeV}$ and $f_{\pi}=0.132 \mathrm{GeV}$. We also choose the cutoff parameter $\Lambda=0.6-1.0 \mathrm{GeV}$, because in the chiral perturbation theory, the momentum transfer is usually less than $1.0 \mathrm{GeV}$.

In the $\Lambda_{c}^{+}-\bar{\Lambda}_{c}^{+}$system, the averaged mass difference between $\Sigma_{c}^{*}$ and $\Lambda_{c}$ is about $\Delta=$ $0.234 \mathrm{GeV}$. The resultant potentials for the spin-singlet and spin-triplet $\Lambda_{c}-\bar{\Lambda}_{c}$ states are plotted in Figs.(4) and (5), respectively. From these figures, we see that comparing with
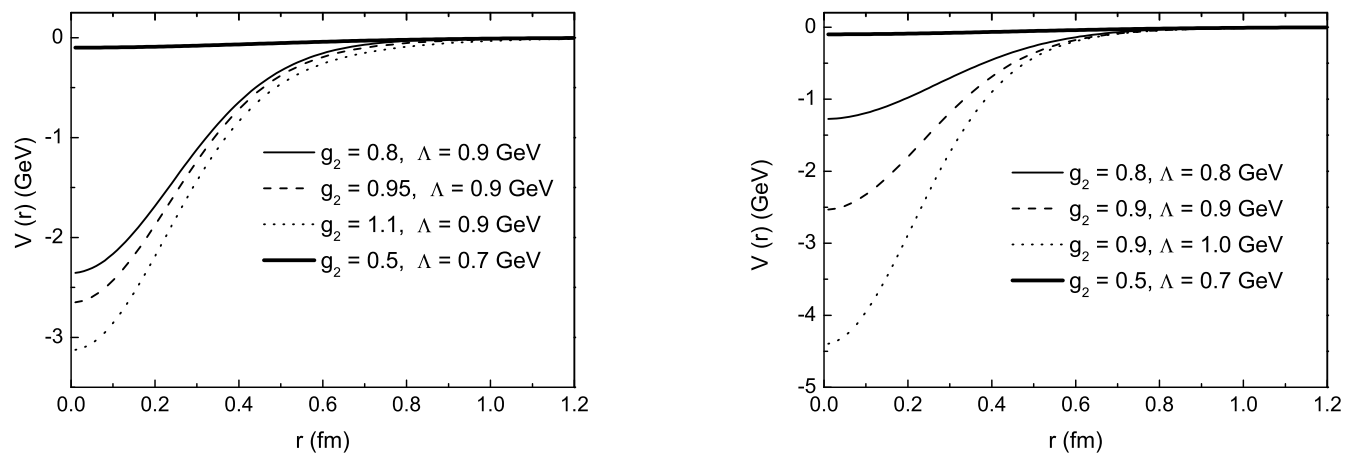

Figure 4: The $\Lambda_{c}-\bar{\Lambda}_{c}$ potential in the singlet state with different $g_{2}$ but fixed $\Lambda$ (left figure) and different $\Lambda$ but fixed $g_{2}$ (right figure).

our previous result [8], no matter in which states, the singular behavior of the potential around origin is greatly reduced. This indicates that the contribution from large $\lambda$ values is also important in the two-pion exchange process. Moreover, the potentials become more attractive with increasing values of $g_{2}$ and $\Lambda$. This is reasonable, because the larger $g_{2}$ value provides stronger coupling and consequently stronger potential. And the value of the cut-off $\Lambda$ largely affects the depth of the potential, the smaller value of $\Lambda$ makes the shorter distance interaction even more suppressed. It partly prevents the $\Lambda_{c}$ and $\bar{\Lambda}_{c}$ getting too close, thus matches our treatment of omitting the $s$-channel interaction. The line shape of these potentials also tells us that the interaction between $\Lambda_{c}$ and $\bar{\Lambda}_{c}$ is attractive and might bind these particles together.

With these potentials, we can study the $\Lambda_{c}-\bar{\Lambda}_{c}$ scattering property. The partial wave 

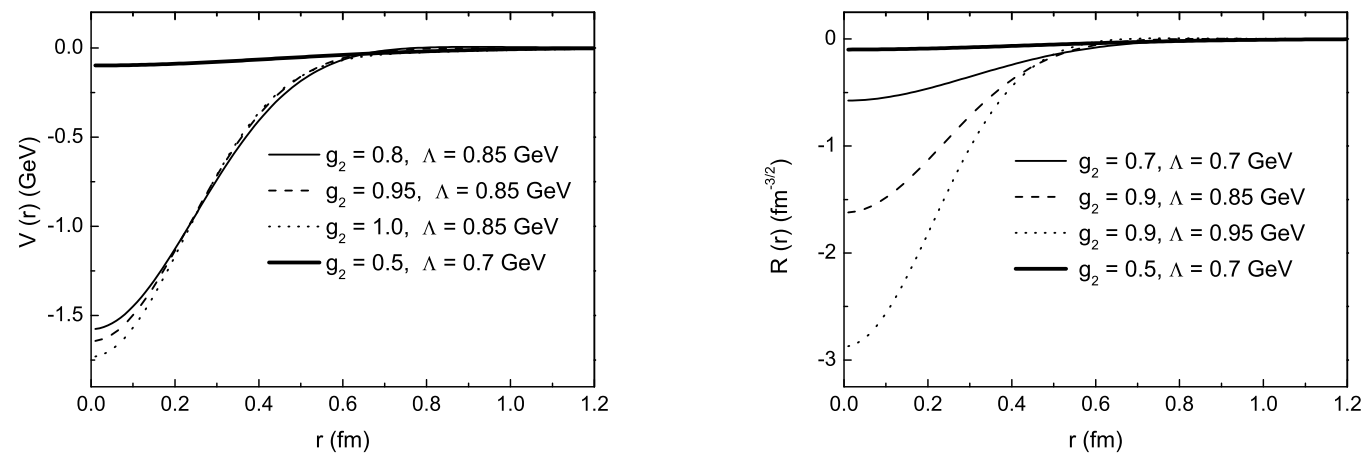

Figure 5: The $\Lambda_{c}-\bar{\Lambda}_{c}$ potential in the triplet state with different $g_{2}$ but fixed $\Lambda$ (left figure) and different $\Lambda$ but fixed $g_{2}$ (right figure).

Schrödinger equation that the $\Lambda_{c}-\bar{\Lambda}_{c}$ scattering obeys reads

$$
\frac{d^{2} u_{l}(r)}{d r^{2}}+\left[k^{2}-\frac{l(l+1)}{r^{2}}-U(r)\right] u_{l}(r)=0,
$$

with the boundary condition

$$
u_{l}(r)=k r j_{l}(k r)+\int_{0}^{\infty} G_{l}\left(r, r^{\prime}\right) U\left(r^{\prime}\right) u_{l}\left(r^{\prime}\right) d r^{\prime}
$$

where $G_{l}\left(r, r^{\prime}\right)$ is the Green function in the form of

$$
\begin{aligned}
G_{l}\left(r, r^{\prime}\right) & =k r r^{\prime} j_{l}(k r) n_{l}\left(k r^{\prime}\right), & & r \leq r^{\prime} \\
& =k r r^{\prime} j_{l}\left(k r^{\prime}\right) n_{l}(k r), & & r \geq r^{\prime}
\end{aligned}
$$

and $U(r)=2 \mu V(r), j_{l}(k r)$ and $n_{l}(k r)$ are the spherical Bessel function and the spherical Neumann function, respectively [17]. Then, the scattering phase shift $\delta_{l}(k)$ and the potential $V(r)$ has the relation

$$
\tan \delta_{l}(k)=-\int_{0}^{\infty} r^{\prime} j_{l}\left(k r^{\prime}\right) U\left(r^{\prime}\right) u_{l}\left(r^{\prime}\right) d r^{\prime} .
$$

Solving above equations numerically, we obtain scattering phase shifts and plot them in Fig,6. From this figure, one sees that although all the potentials are attractive, in some cases, the potential does not support a binding character, especially in the case where the value of $g_{2}$ is extracted from the $\Sigma_{c} \rightarrow \Lambda_{c} \pi$ decay data (the thick solid curve). It means 

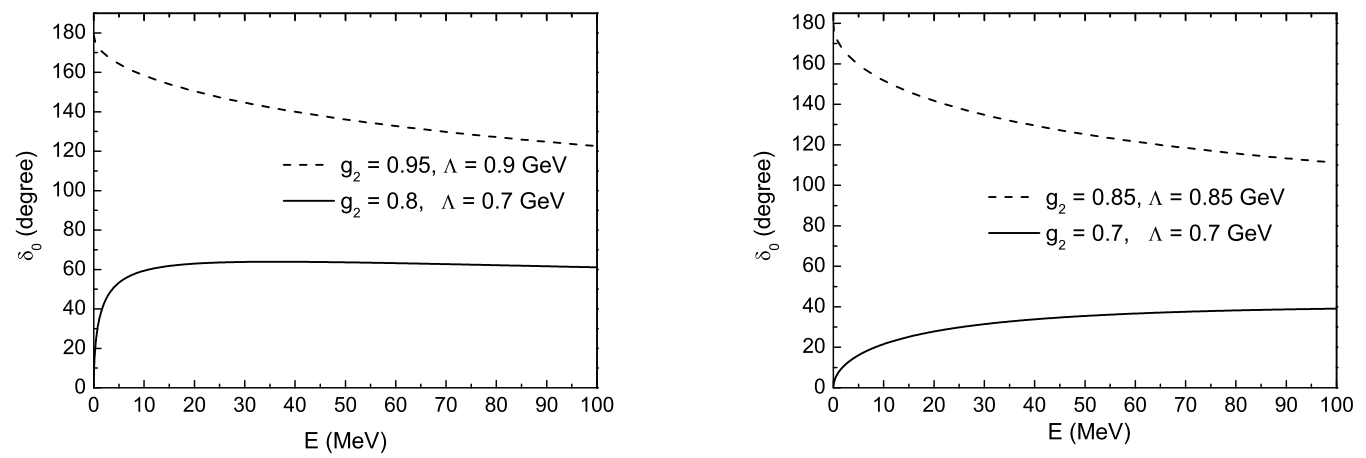

Figure 6: Phase shifts of the $\Lambda_{c}-\bar{\Lambda}_{c}$ system in the spin-singlet (left figure) and the spintriplet (right figure) states.

that the $\Lambda_{c}-\bar{\Lambda}_{c}$ could be bound (the dashed curve) only when the coupling constant $g_{2}$ takes a value larger than that from the data fitting, and the cut-off $\Lambda$ is larger than that in the light baryon sector.

Moreover, we can also calculate the scattering length for concerned states by

$$
a=-\lim _{k \rightarrow 0} \frac{\tan \delta_{0}(k)}{k}
$$

The results are tabulated in Table 1.

Table 1: Scattering length for the spin-singlet and spin-triplet states in the $\Lambda_{c^{-}} \bar{\Lambda}_{c}$ and $\Sigma_{c^{-}}$ $\bar{\Sigma}_{c}$ systems.

\begin{tabular}{cc||cc}
\hline \hline \multicolumn{2}{c||}{$S=0$ state for $\Lambda_{c}-\bar{\Lambda}_{c}$} & \multicolumn{3}{c}{$S=1$ state for $\Lambda_{c}-\bar{\Lambda}_{c}$} \\
\hline$g_{2}=0.95, \Lambda=0.9 \mathrm{GeV}$ & $a=3.5 \mathrm{fm}$ & $g_{2}=0.85, \Lambda=0.85 \mathrm{GeV}$ & $a=2.1 \mathrm{fm}$ \\
$g_{2}=0.8, \Lambda=0.7 \mathrm{GeV}$ & $a=-2.7 \mathrm{fm}$ & $g_{2}=0.7, \Lambda=0.7 \mathrm{GeV}$ & $a=-0.6 \mathrm{fm}$ \\
\hline \hline \multicolumn{2}{c||}{$S=0$ state for $\Sigma_{c}-\bar{\Sigma}_{c}$} & \multicolumn{2}{c}{$S=1$ state $\Sigma_{-} \bar{\Sigma}_{c}$} \\
\hline$g_{1}=0.85, \Lambda=1.1 \mathrm{GeV} \quad a=3.5 \mathrm{fm}$ & $g_{1}=0.95, \Lambda=1.1 \mathrm{GeV}$ & $a=5.7 \mathrm{fm}$ \\
$g_{1}=0.8, \Lambda=0.8 \mathrm{GeV}$ & $a=-1.7 \mathrm{fm}$ & $g_{1}=0.8, \Lambda=0.8 \mathrm{GeV}$ & $a=-0.8 \mathrm{fm}$ \\
\hline \hline
\end{tabular}

The scattering lengths in this table also tell us that only those $g_{2}$ and $\Lambda$ values, with which the attractive potential is much stronger (denoted by dashed curve in Figs 445), can 
produce an appropriate positive scattering length, which denotes a bound $\Lambda_{c}-\bar{\Lambda}_{c}$ system, otherwise the system is unbound.

Based on the enlightenment from the scattering study, we further perform the bound state calculation to check the condition for forming a $\Lambda_{c}-\bar{\Lambda}_{c}$ bound state. Since we have the spin-spin interaction in the $\Lambda_{c}-\bar{\Lambda}_{c}$ potential Eq.(39), substituting such a potential into the Schrödinger equation and solving the equation numerically, we get the binding energy for the spin splitted $S=0$ and $S=1$ states, respectively. The results are tabulated in Table 2.

Table 2: Binding energies (BE), as well as the masses of heavy baryonium $\left(M_{\Lambda_{c} \bar{\Lambda}_{c}}\right)$, in the $\Lambda_{c}-\bar{\Lambda}_{c}$ system in various parameter cases.

\begin{tabular}{cccc||cccc}
\hline \hline \multicolumn{3}{c||}{$S=0$ state } & \multicolumn{4}{c}{$S=1$ state } \\
$\left|g_{2}\right|$ & $\Lambda(\mathrm{GeV})$ & $\mathrm{BE}(\mathrm{MeV})$ & $M_{\Lambda_{c} \bar{\Lambda}_{c}}(\mathrm{GeV})$ & $\left|g_{2}\right|$ & $\Lambda(\mathrm{GeV})$ & $\mathrm{BE}(\mathrm{MeV})$ & $M_{\Lambda_{c} \bar{\Lambda}_{c}}(\mathrm{GeV})$ \\
\hline$<0.8$ & $<0.8$ & - & - & $<0.7$ & $<0.7$ & - & - \\
0.9 & 0.9 & 34 & 4.538 & 0.9 & 0.85 & 75 & 4.497 \\
0.9 & 1.0 & 118 & 4.45 & 0.9 & 0.95 & 285 & 4.287 \\
\hline 0.8 & 0.9 & 3.25 & 4.568 & 0.8 & 0.85 & 14 & 4.558 \\
1.1 & 0.9 & 166.2 & 4.406 & 1.0 & 0.85 & 199 & 4.373 \\
\hline \hline
\end{tabular}

From this table, we see that with the extracted $g_{2}$ value of $0.5 \sim 0.57$ from the decay data, the $\Lambda_{c}-\bar{\Lambda}_{c}$ system would not be bound. If we wish $\Lambda_{c}$ and $\bar{\Lambda}_{c}$ being bound, no matter in the spin singlet state or the spin triplet state, the coupling constant should be much larger than the value extracted phenomenologically, namely $g_{2}>0.8$ for the spin-singlet state and $g_{2}>0.78$ for the spin-triplet state. This is in coincidence with those learned from above scattering study. The result also shows the required ranges of $g_{2}$ and $\Lambda$ for $\Lambda_{c}-\bar{\Lambda}_{c}$ binding: $0.8<g_{2} \leq 1.1$ and $0.8 \mathrm{GeV}<\Lambda \leq 1.0 \mathrm{GeV}$ for the spin singlet state and $0.7<g_{2} \leq 1.0$ and $0.7 \mathrm{GeV}<\Lambda \leq 0.95 \mathrm{GeV}$ for the spin triplet state, respectively. The mass of the corresponding baryonium is in the region of $(4.406,4.572] \mathrm{GeV}$ and (4.287, 4.572] GeV for the spin-singlet and spin-triplet states, respectively.

Same calculations can be performed for the $\Sigma_{c}-\bar{\Sigma}_{c}$ system as well. The potentials for the spin-singlet and spin-triplet states are plotted in Fig:7 and Fig [8, respectively. From 

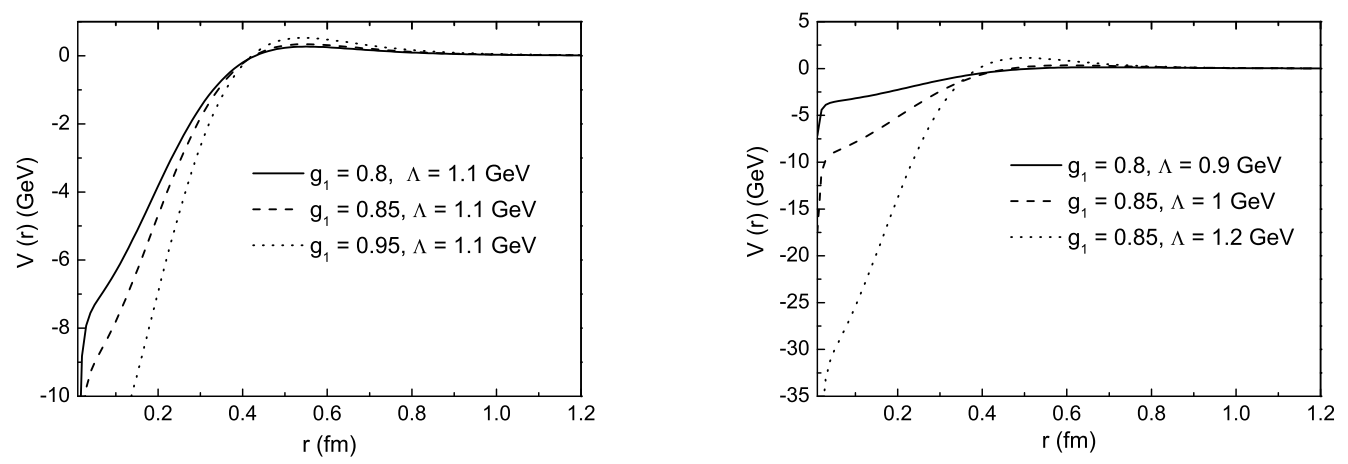

Figure 7: $\Sigma_{c^{-}} \bar{\Sigma}_{c}$ potential in spin-singlet state.
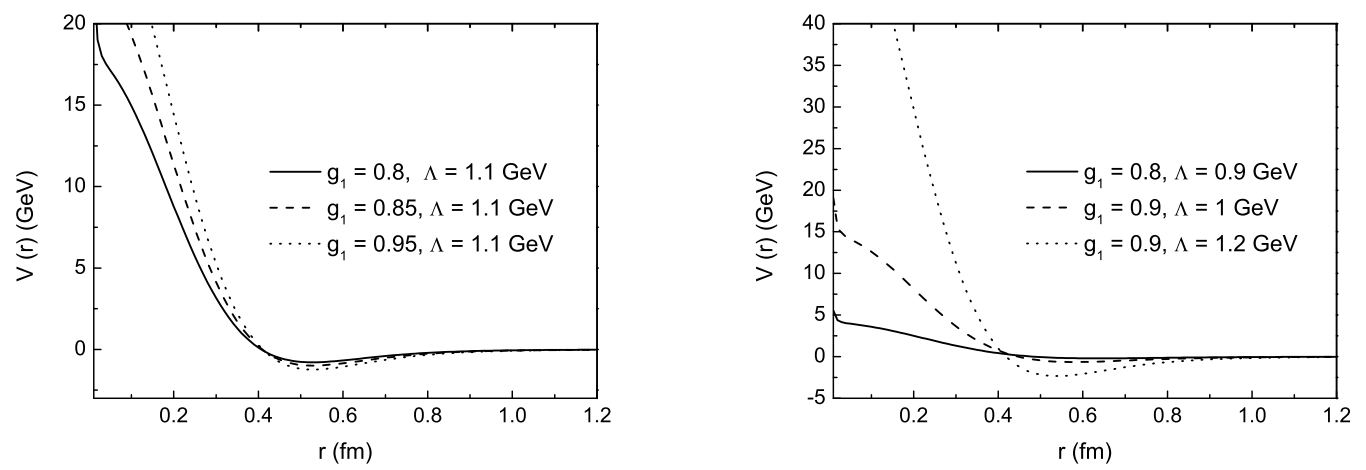

Figure 8: $\Sigma_{c^{-}} \bar{\Sigma}_{c}$ potential in spin-triplet state. 
these figures, it is shown that the potentials between $\Sigma_{c}$ and $\bar{\Sigma}_{c}$ are different from those between $\Lambda_{c}$ and $\bar{\Lambda}_{c}$, especially in the spin-triplet state where the potential has a repulsive core in the short distance. This is due to the contribution from the one-pion exchange, which gives the spin-spin interaction, in the $\Sigma_{c^{-}} \bar{\Sigma}_{c}$ interaction. The phase shifts of the $\Sigma_{c^{-}}$ $\bar{\Sigma}_{c}$ system are plotted in Fig, 9, Again, the system in some cases could be bound (dashed
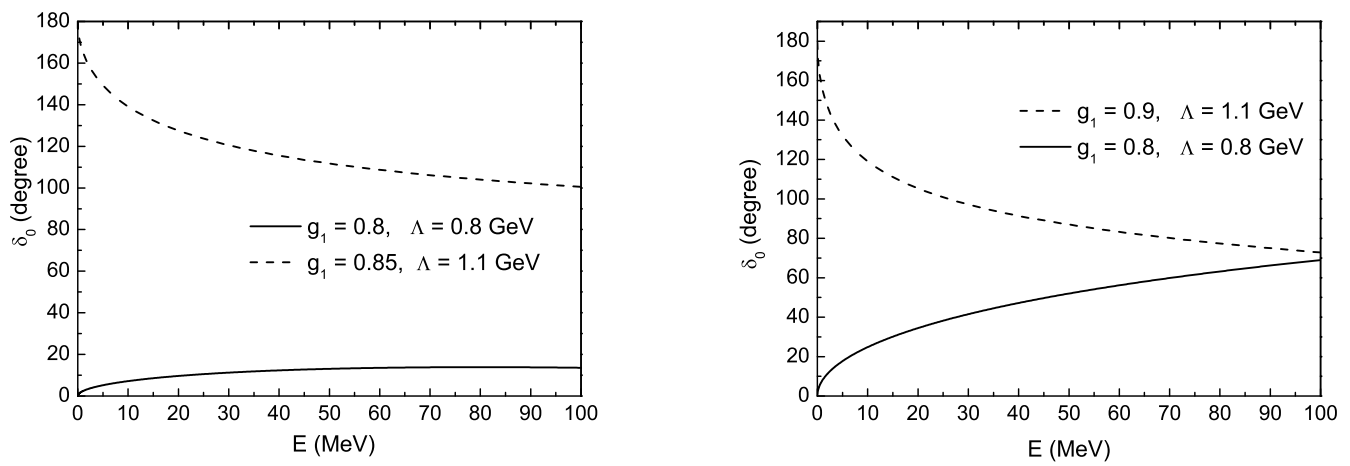

Figure 9: Phase shifts of the $\Sigma_{c}-\bar{\Sigma}_{c}$ system in the spin-singlet (left figure) and the spintriplet (right figure) states.

curve) and in the other cases would be unbound (solid curve). However, due to lack of experimental data to fix the $g_{1}$ value, it is necessary to examine the marginal condition for its binding.

Same as before, substituting the obtained $\Sigma_{c}-\bar{\Sigma}_{c}$ potential, Eq.(47), into the Schrödinger equation and solving it numerically, we have the binding character for the $\Sigma_{c}-\bar{\Sigma}_{c}$ system. The resultant binding energies for the $S=0$ and $S=1$ states are tabulated in Table3.

From this table, we find that as long as $g_{1}>0.8$ and $\Lambda>1.0 \mathrm{GeV}$ in the spin-singlet state and $g_{1}>0.9$ and $\Lambda>1.0 \mathrm{GeV}$ in the spin-triplet state, the $\Sigma_{c}-\bar{\Sigma}_{c}$ system could be bound. And also the spin-triplet state is slightly easier to be bound than the spintriplet state. The result also shows the required ranges of $g_{1}$ and $\Lambda$ for the $\Sigma_{c}-\bar{\Sigma}_{c}$ binding: $0.8<g_{2} \leq 0.85$ and $0.95 \mathrm{GeV}<\Lambda \leq 1.1 \mathrm{GeV}$ for the spin singlet state and $0.8<g_{2} \leq 0.9$ and $0.95 \mathrm{GeV}<\Lambda \leq 1.1 \mathrm{GeV}$ for the spin triplet state, respectively. The mass of the corresponding baryonium is in the region of $(4.880,4.910]$ and $(4.870,4.910] \mathrm{GeV}$ for the spin-singlet and spin-triplet states, respectively. 
Table 3: Binding energies (BE), as well as the masses of heavy baryonium $\left(M_{\Sigma_{c} \bar{\Sigma}_{c}}\right)$, for the $\Sigma_{c^{-}} \bar{\Sigma}_{c}$ system.

\begin{tabular}{cccc||cccc}
\hline \hline & \multicolumn{3}{c||}{$S=0$ state } & \multicolumn{4}{c}{$S=1$ state } \\
$\left|g_{1}\right|$ & $\Lambda(\mathrm{GeV})$ & $\mathrm{BE}(\mathrm{MeV})$ & $M_{\Sigma_{c} \bar{\Sigma}_{c}}(\mathrm{GeV})$ & $\left|g_{1}\right|$ & $\Lambda(\mathrm{GeV})$ & $\mathrm{BE}(\mathrm{MeV})$ & $M_{\Sigma_{c} \bar{\Sigma}_{c}}(\mathrm{GeV})$ \\
\hline$<0.8$ & $<0.95$ & - & - & $<0.8$ & $<0.95$ & - & - \\
0.85 & 1 & 14 & 4.896 & 0.9 & 1 & 4.5 & 4.9 \\
\hline 0.8 & 1.1 & 21.7 & 4.89 & 0.8 & 1.1 & 12.7 & 4.897 \\
0.85 & 1.1 & 29 & 4.88 & 0.85 & 1.1 & 39.9 & 4.87 \\
\hline \hline
\end{tabular}

The similar study can be done for the systems with the bottom flavor. In the $\Lambda_{b}^{+}-\bar{\Lambda}_{b}^{+}$ system, the averaged mass difference between $\Sigma_{b}^{*}$ and $\Lambda_{b}$ is about $\Delta=0.114 \mathrm{GeV}$. With the same reason in the charm flavor sector, namely due to lack of the experimental data to fix the $g_{b}$ value, we also examine the marginal condition for its binding. Carrying out the same procedure, we obtain the binding character of the $\Lambda_{b}-\bar{\Lambda}_{b}$ system. The resultant binding energies in the $S=0$ and $S=1$ states are tabulated in Table4. The result shows

Table 4: Binding energies (BE), as well as the masses of heavy baryonium $\left(M_{\Lambda_{b} \bar{\Lambda}_{b}}\right)$, in the $\Lambda_{b}-\bar{\Lambda}_{b}$ system.

\begin{tabular}{cccc||cccc}
\hline \hline \multicolumn{3}{c||}{$S=0$ state } & \multicolumn{4}{c}{$S=1$ state } \\
$\left|g_{b}\right|$ & $\Lambda(\mathrm{GeV})$ & $\mathrm{BE}(\mathrm{MeV})$ & $M_{\Lambda_{b} \bar{\Lambda}_{b}}(\mathrm{GeV})$ & $\left|g_{b}\right|$ & $\Lambda(\mathrm{GeV})$ & $\mathrm{BE}(\mathrm{MeV})$ & $M_{\Lambda_{b} \bar{\Lambda}_{b}}(\mathrm{GeV})$ \\
\hline$<0.65$ & $<0.8$ & - & - & $<0.55$ & $<0.85$ & - & - \\
0.8 & 0.85 & 25.5 & 11.21 & 0.6 & 0.85 & 4.1 & 11.23 \\
\hline 0.68 & 0.8 & 7.5 & 11.23 & 0.55 & 0.9 & 8 & 11.23 \\
0.7 & 0.9 & 15.6 & 11.22 & 0.6 & 0.9 & 38.5 & 11.2 \\
\hline \hline
\end{tabular}

that for the $\Lambda_{b}-\bar{\Lambda}_{b}$ system, a relatively smaller $g_{b}$ value can make the spin-triplet state bound. The result also presents the required ranges of $g_{b}$ and $\Lambda$ for the $\Lambda_{b}-\bar{\Lambda}_{b}$ binding: $0.65<g_{b} \leq 0.8$ and $0.8 \mathrm{GeV} \leq \Lambda \leq 0.9 \mathrm{GeV}$ for the spin-singlet state and $0.55<g_{b} \leq 0.6$ and $0.85 \mathrm{GeV} \leq \Lambda \leq 0.9 \mathrm{GeV}$ for the spin-triplet state, respectively. The mass of the corresponding baryonium is in the region of $(11.21,11.24]$ and $(11.2,11.24] \mathrm{GeV}$ for the spin-singlet and spin-triplet states, respectively. 
We also notice that someone has calculated the value of $g_{b}$ [18, 19], recently. They give $\left|g_{b}\right|=0.475 \pm 0.050$ for the $\Sigma_{b}^{*}-\pi-\Lambda_{b}$ coupling. This value seems too small to support a bound $\Lambda_{b}-\bar{\Lambda}_{b}$ state. However, the final conclusion should not be made before some issues are clarified, like, whether or not the $\Sigma_{b}^{*}-\pi-\Lambda_{b}$ coupling can be straightforwardly applied to the loop calculation. When future decay data of the $b$-flavored baryon become available, we would be able to extract a physical value of $g_{b}$. If the extracted $g_{b}$ is consistent with the marginal $g_{b}$ value for binding in this calculation, one might confirm such a $b$-flavored heavy-baryonium.

\section{Conclusion}

The heavy-baryon-anti-heavy-baryon systems are studied in the framework of heavy baryon chiral perturbation theory. The potentials for the $\Lambda_{c}-\bar{\Lambda}_{c}, \Sigma_{c}-\bar{\Sigma}_{c}$ and $\Lambda_{b}-\bar{\Lambda}_{b}$ interactions are derived with the two-pion exchange mechanism. Unlike our previous work, we use the holonomic potential to investigate the scattering and binding characters in this paper. The scattering characters of these systems are calculated by solving the partial Schrödinger equation. From the obtained phase shifts and the scattering lengths, it is found that the $\Lambda_{c}-\bar{\Lambda}_{c}$ system could be bound with a $g_{2}$ value larger than that extracted phenomenologically from the decay data of charmed baryons or estimated by Ref.[13].

For the $\Sigma_{c}-\bar{\Sigma}_{c}$ system, since we do not have available decay data to fix $g_{1}$, whether the system is bound depends on the selected value of $g_{1}$. To confirm these results, the bound state calculations are further performed. It is shown that marginal $g_{2}$ value for binding is about 0.8 which is larger than the physical value of about $0.5 \sim 0.57$. In the $\Sigma_{c}-\bar{\Sigma}_{c}$ system, the marginal $g_{1}$ value for binding is also estimated. The minimum $g_{1}$ value is about 0.85 . This value should be compared with that extracted from the future data to affirm whether the $\Sigma_{c}-\bar{\Sigma}_{c}$ system could be bound. The $\Lambda_{b}-\bar{\Lambda}_{b}$ system is studied as well. It is found that the minimum $g_{b}$ value for binding is much smaller than that for the $\Lambda_{c}-\bar{\Lambda}_{c}$ system. If the $g_{b}$ value extracted from the future decay data of the $b$-flavored baryon can meet this value, one might confirm such a $\Lambda_{b}-\bar{\Lambda}_{b}$ heavy baryonium.

It should be mentioned that above conclusions are also related the cutoff value which 
is assumed to be similar to that for the light hadron sector in chiral perturbation theory. A similar situation was met in Ref.[20], where a relative large cutoff is also required for a possible molecule state in the $\Lambda_{c}-\Lambda_{c}$ system in the one-pion-exchange model. Furthermore, it is worthwhile to emphasize that in order to more realistically affirm whether the heavybaryon-anti-heavy-baryon system could have a bound state, namely a heavy baryonium, the annihilation channel and couple channel effects on the heavy baryonium potential should also be taken into account. In particular, a study in the quark-gluon degree of freedom is necessary.

Even some corrections should be further considered, our results are much more reliable and stable than those in our early calculation [8]. From the regions of possible heavy baryonium masses, we conjecture that up to this stage, $Y(4260)$ and $Y(4360)$ could be a spin-triplet $\Lambda_{c}-\bar{\Lambda}_{c}$ baryonium, but $Y(4660)$ could not be a $\Lambda_{c}-\bar{\Lambda}_{c}$ baryonium in either spin-singlet or spin-triplet state, and $Y(10890)$ could not be a $\Lambda_{b}-\bar{\Lambda}_{b}$ baryonium either. Moreover, because $Z^{ \pm}(4430)$ is a electrically changed state and $Z_{c}(3900)$ is out of the possible binding range, they are nothing to do with the $\Lambda_{c}-\bar{\Lambda}_{c}$ baryonium.

\section{Acknowledgments}

This work was supported in part by the National Natural Science Foundation of China(NSFC) under the grants 10935012, 10821063, 11175249, 11035006 and 11165005. 


\section{References}

[1] S. K. Choi et al. (Belle Collaboration), Phys. Rev. Lett. 91, 262001 (2003); B. Aubert et al. (BaBar Collaboration), Phys. Rev D 77,111101 (2008); K. F. Chen et al. (Belle Collaboration), Phys. Rev. Lett.100,112001 (2008).

[2] M.Ablikim et al. [BESIII Collaboration], Phys.Rev.Lett. 110 , 252001(2013).

[3] Z. Q. Liu et al. (Belle Collaboration), Phys. Rev. Lett. 110, 252002 (2013).

[4] N.Brambilla, et al. Heavy quarkonium: progress, puzzles, and opportunities, Euro. Phys. J. C 71, 1534 (2011), (and references therein).

[5] Dian-Yong Chen, Xiang Liu and Takayuki Matsuki, Phys.Rev.Lett. 110, 232001 (2013); arXiv:1304.5845 [hep-ph].

[6] Feng-Kun Guo, Carlos Hidalgo-Duque, Juan Nieves and Manuel Pavon Valderrama, arXiv:1303.6608 [hep-ph].

[7] Jian-Rong Zhang, Phys. Rev. D87, 116004 (2013).

[8] Y.-D. Chen and C.-F. Qiao, Phys, Rev D 85, 034034 (2012) (and references therein).

[9] C.-F. Qiao, J. Phys. G: Nucl. Part. Phys. 35, 075008 (2008); C.-F. Qiao, Phys. Lett. B 639, 263 (2006).

[10] K. G. Richardson, Chiral Symmetry and the Nucleon-Nucleon Interaction, arXiv :0008118 [hep-ph].

[11] A. Manohar and H. Georgi, Nucl. Phys. B 234, 189 (1984).

[12] Mark B. Wise, Phys. Rev. D 45, R2188 (1992)

[13] Tung-Mow Yan, Y. C. Lin and Hoi-Lai Yu, Phys. Rev D46, 1148 (1992); Hai-Yang Cheng, Tung-Mow Yan et al. Phys. Rev D47, 1030 (1993).

[14] Th. A. Rijken and V. G. J. Stoks, Phys. Rev. C46, 73 (1992); Th.A. Rijken and V. G. J. Stoks, Phys. Rev. C46, 102 (1992); 
[15] Th.A. Rijken, Ann. Phys. 208, 253 (1991).

[16] K. Nakamura, et al.(Particle Date Group), J. Phys. G: Nucl. Part. Phys. 37, 1 (2010).

[17] M. S. Stern, Comput.Phys.Commun. 17, 365 (1979).

[18] William Detmold, C.-J. David Lin, Stefan Meinel, Phys. Rev. Lett 108.172003 (2012)

[19] William Detmold, C.-J. David Lin, Stefan Meinel, Phys. Rev. D85,114508 (2012).

[20] Wakafumi Meguro, Yan-Rui Liu and Makoto Oka, Phys. Lett B704, 547 (2011). 